\title{
Using Sound Patterns to Enhance Directional Sound for Emergency Route Guidance
}

\author{
Tom Plocher ${ }^{1}$, Zhaoxia Janet Jin ${ }^{2}$, and Foong-Yeen Donny Chan ${ }^{2}$ \\ ${ }^{1}$ Honeywell International, Automation and Control Solutions, Advanced Technology \\ Laboratory, 1985 Douglas Drive, Golden Valley, MN 55422 USA \\ ${ }^{2}$ Honeywell Technology Solutions-China, 430 Li Bing Rd, Zhangjiang Hi-Tech Park, \\ Shanghai, 201203 China
}

\begin{abstract}
Broadband sound emitted by digital sounder devices has been shown be a useful means for guiding building occupants to the correct emergency, particularly under conditions of darkness and obscuration from smoke. Standard practice has been to place a single sounder by the emergency exit door to function as an "auditory exit sign." The current paper reports on studies conducted in full scale building environments that investigated ways in which multiple sounders could be used along a route in a building to provide emergency route guidance to building occupants. The relative effectiveness of various patterns of sounder activation patterns is described. One such pattern that uses sequential activation of sounders together with number of sound pulses to encode sounder position along the evacuation route is particularly promising.
\end{abstract}

Keywords: directional sound, guidance, audio, evacuation, buildings.

\section{Introduction}

Withington $[1,2,3]$ was the first to demonstrate the practical use of directional broadband sound as a means to guide building occupants to the correct emergency exit. Withington used a device that generated a locating sound comprised of a majority of the frequencies in the human hearing range emitted simultaneously. The result is a "shhhhhh" sound that is readily localized by humans. Withington's concept placed a single directional sounder device at each exit. Sounding the device attracted people in the vicinity to the nearest exit. Thus, essentially it was an audio version of the visual Exit signs commonly seen above exit doors in North American buildings. However, directional sound has been shown to be effective even in conditions of darkness or heavy smoke obscuration, conditions in which Exit signs are not visible or useful.

Withington's initial concept used single directional sounders placed at exit doors and were aimed at assisting evacuations in relatively small building spaces. However, more advanced concepts, involving many directional sounders arrayed at 10-15 meter intervals and emitting sound in a coordinated manner have been proposed as a means for actually guiding building occupants down a safe route of evacuation. These advanced route guidance concepts are promising, particularly when combined with new models for predicting the spread of smoke associated with a fire and hence, identifying safe evacuation routes. 
The research reported here was conducted to understand how to enhance current practice in coordinating multiple directional sounders to create sound patterns that are effective for route guidance in both small and large building spaces.

\section{Pilot Tests}

Initially, pilot tests were conducted in order to better understand how humans recognize and respond to directional sound patterns. The issues identified by the pilot tests then became the focus of a more formal and larger scale experiment.

\subsection{Method}

Directional Sound Device. All of the pilot tests and the experiment reported here used a directional sounder device called ExitPoint ${ }^{\mathrm{TM}}$, marketed by Honeywell International, Inc. This device has an integral audio amplifier that produces a pulsating sound consisting of broadband low, mid, and high range sounds. In addition to the broadband noise, the sounder is capable of playing an alert message in the form of a recorded voice message or other audible signals. The device, shown in Figure 1, comes from the factory pre-programmed with the four alternative pulse patterns shown in Table 1. The concept of use is to array the sounders along an evacuation route, setting each sounder to a different pulse rate, from Slow for the first sounder along the route, to Mid2 to Mid1, and finally to a Fast rate at the end of the route, e.g. the emergency exit door. Examples of simple sounder arrays are shown in Figures 2 and 3.

Table 1. Original pulse patterns

\begin{tabular}{|l|l|l|}
\hline & $\begin{array}{l}\text { Duration } \\
\text { ON } \\
\text { (msec) }\end{array}$ & $\begin{array}{l}\text { Duration } \\
\text { OFF } \\
\text { (msec) }\end{array}$ \\
\hline Slow & 300 & 400 \\
\hline Mid2 & 225 & 200 \\
\hline Mid1 & 225 & 100 \\
\hline Fast & 150 & 50 \\
\hline
\end{tabular}

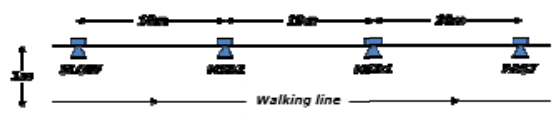

Fig. 2. Straight line sounder arrangement

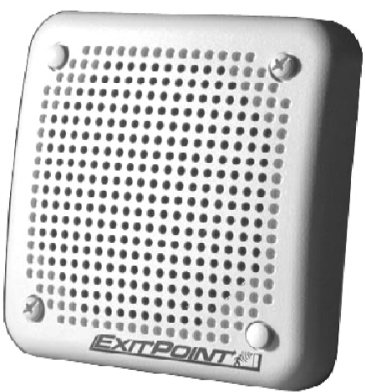

Fig. 1. ExitPoint ${ }^{\mathrm{TM}}$ sounder device

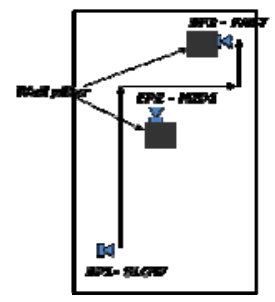

Fig. 3. "S" Shape Arrangement with sounders placed at turns in corridor 
Our first pilot test observations were conducted in a public cafeteria of dimensions $50 \mathrm{~m} \times 15 \mathrm{~m}$. It was a highly reverberant environment, with tiled floor and a combination of glass brick walls. The ambient environmental noise measured at $67 \mathrm{~dB}$ owing to people at work and air-conditioning noise. The sounders were set to emit sound at $1 / 4$ watt power and were placed at regular $10 \mathrm{~m}$ intervals along the routes. Each sounder was set at a different pulse rate and, in terms of pulse rate, were arrayed from Slow to Medium 2 to Medium 1 to Fast, along the route. In some trials, all sounders were activated simultaneously throughout the trial. In other trials, only two adjacent sounders were activated simultaneously at any given time. On some trials, subjects were instructed to walk in a straight line from Slow to Fast as in Figure 2. In other trials, they were instructed to walk from Slow to Fast along a route laid out in an "S" shape as in Figure 3. Subjects' behavior was recorded on video and they were debriefed about their experience after the trials. Six subjects participated in these initial pilot trials.

These initial limited pilot trials suggested that mutual interference between adjacent sounders potentially was a problem and deserved further investigation. The initial trials also suggested that we needed a larger and more complicated test environment to represent a more typical environment in which sounders would be used. Thus, a second series of pilot test trials were conducted to discover how to make each individual sounder easier to discriminate from the sounders adjacent to it. Two deployment concepts were set up and compared:

- Single Exit Sounder- A single sounder set to Fast and also emitting an audible message, "Exit Here", was placed at the exit door at the end of the evacuation route. No other sounders were used.

- "Sounder All @ Once"- In this concept, four sounders were placed along the route from Slow to Mid2 to Mid1 to Fast at the exit door, activated simultaneous1y. In some trials, the Off component of the pulse rate for the Mid2 sounder was changed from its original factory setting of $200 \mathrm{msec}$ to a new value of $300 \mathrm{msec}$ in an attempt to make it more distinct from the Mid1 sounder and better guide the subjects through the middle portion of the route. As in the first concept, the Fast sounder at the exit door also emitted an audible "Exit Here" message.

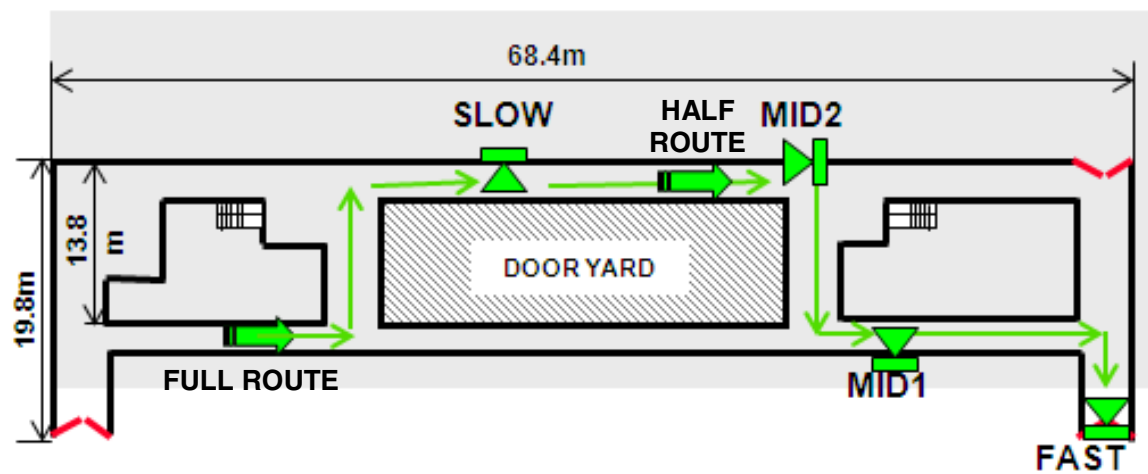

Fig. 4. Test environment for second pilot test 
Pilot Test Protocol. Twenty-four college students with normal hearing participated. The larger and more complicated test environment shown in Figure 4 was used, a single floor of a university class building with typical concrete and glass construction. Subjects were instructed that their task was to move along an evacuation route to an exit door by following the sounders. On each trial, the subject started from either of the two starting points shown in Figure 4 by the bold green arrows. One starting point, noted as "Full Route" in the figure, was at the very beginning of the route and well ahead of the first sounder in the sequence. The subject's task was to identify and move to the first sounder (the Slow one) and then on along the route. The distance of travel along the full route was 83.5 meters. The other or "Half Route" starting point shown in the figure was in between the first and second sounders in the sequence. In these half route trials, the subject's initial task was slightly different. He or she first had to determine which direction to go, move toward the correct sounder (the Mid2 in this case) and then on along the route. This simulated the very real case that an evacuee might not always evacuate from the beginning of the route, but might just as readily find himself or herself starting somewhere in the middle and immediately facing a choice of which direction to head. The distance of travel along the half route was 46.8 meters.

Subjects wore half-blindfolds to simulate an environment filled with smoke, limiting visibility to less than 1 meter. After donning the blindfold before each trial started, the subject was lead around the corridors of the space in order to reduce any spatial memory of building layout. Barriers with alarm flags were used to show "danger here!" and prevent participants from wandering outside the planned test route.

\subsection{Pilot Test Findings}

Our pilot test observations highlighted a number of issues and potential deployment concepts for enhancing the effectiveness of the sounders for route guidance:

- Single Sounders- Single sounders were easy to hear and locate in the cafeteria setting of the first pilot test in which the user was within audible range of the single sounder all the time. In this situation the single sounder provided effective guidance. Single sounders were less useful in the larger university building setting of the second pilot test. In this larger setting, of course, a single exit sounder cannot provide selective guidance when alternate routes to the exit are possible. Also, users reported that they felt confused and scared when they were outside of the single sounder's audible range and couldn't hear the voice message "Exit Here".

- Sounder All At Once Concept- This concept was able to provide route guidance in the large classroom/office building environment of the second pilot test. Subjects could hear guidance cues wherever they were along the evacuation route. Subjects reported that it was especially useful in helping them make the correct turn at choice points, where paths crossed each other. However, the pilot tests also revealed some issues with this concept:

- "Single vicinity zone effect"- When subjects were very close to a particular sounder, they could hear only that sounder and not the adjacent ones, causing hesitation about which direction to go next.

- Mutual interference between sounders- Subjects had difficulty discriminating between pulse rates when all four sounders were turned on 
simultaneously and they were in between sounders along the route. They often paused to listen and determine which sounder was dominant and the appropriate one to follow. This hesitation was reflected in their times to traverse the evacuation route, which tended to be slightly longer than when guided by a single sounder. The tradeoff of their hesitation was more correct decisions about the path compared to the single exit sounder trials. Activating only two sounders at a time made each more distinct and appeared to be useful for route guidance.

- Meaning of Fast versus Slow- Some participants confused the meaning of Slow and Fast pulse rates and mistakenly thought that Slow meant safe. Training eliminated this problem.

- Recognizing certain pulse rates- Subjects easily recognized the Slow and Fast pulse rates, but reported difficulty recognizing the Mid2 and Mid1 rates.

- Discriminating between adjacent pulse rates- Subjects readily discriminated between Fast and Slow pulse rates and between Slow and Mid1. But they had difficulty discriminating between sounders that were closely related in pulse rate and adjacent along the route, e.g. between Slow and Mid2 rates, between Mid1 and Fast rates, and between Mid2 and Mid1. Changing the Off component of the pulse rate for the Mid2 sounder from its factory setting of $200 \mathrm{msec}$ to a new value of $300 \mathrm{msec}$ made it more distinct from the Mid1 sounder and provided better guidance to the subjects through the middle portion of the route.

- "Fast" sounder at exit door- A sounder at the exit door, set to Fast and sounding the audible message, "Exit Here" reduced the subject's confusion as long as it was always within clear audible range.

\section{Experiment}

A more formal experiment was conducted to compare the effectiveness of three sounder arrangements: 1) the Single Sounder concept, 2) the Sounder All @ Once concept using the modified wider pulse rate, and 3) a new concept called, Sequential Loop with Redundant Cues. In this new concept, as in the "Sounder All @ Once" concept, the four sounders were placed along the route from Slow to Mid2 to Mid1 to Fast at the exit door. However, this concept differed in three ways. First, in order to reduce mutual interference, the sounders were activated one at a time, in sequence, not simultaneously. When the sequence completed, it returned to the Slow sounder at the beginning and repeated the sequence, as in a loop. Second, the Slow, Mid2 and Mid 1 sounders were set to pulse or "sh" for $250 \mathrm{msec}$ followed by a pause of a minimum of $200 \mathrm{msec}$. The Fast sounder was also set to pulse or "Sh" for $250 \mathrm{msec}$ but each pulse was followed by a pause of only $100 \mathrm{msec}$. It was perceived as pulsing faster. Third, the pulse rates were manipulated so as to provide a redundant audio cue indicating position. Figure 5 illustrates this. Each sounder emitted a unique number of "sh" pulses to indicate its position in the sequence. Thus, the Slow sounder always emitted only one "sh", the Mid2 emitted two "sh" sounds, Mid1 emitted three "sh" sounds, and Fast emitted four "sh" sounds followed by the "Exit Here" message. After each sounder emitted its unique number of pulses, it remained silent until the sequence was complete and looped back to it for another iteration. 


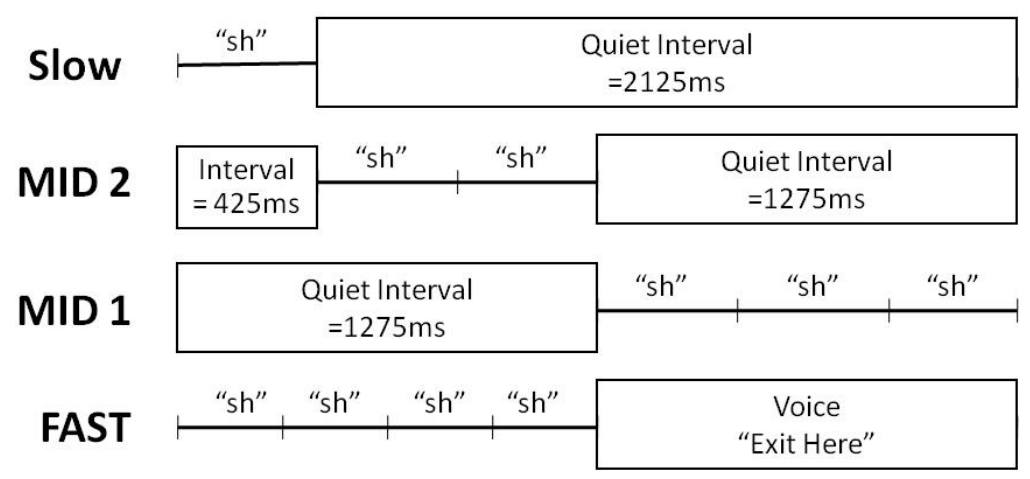

Fig. 5. Sequential loop pattern with redundant cues

\subsection{Method}

Design. A Sound Pattern X Route Within Subjects design was used. There were three sound patterns (Single Sounder, "Sounder All @ Once", Sequential Loop with Redundant Cues) and two routes (Full and Half). The order of treatments for each subject was counterbalanced. Dependent measures were completion (evacuation) time and error rate. Time of Completion was defined as time consumed by moving from the starting location to the sounder at the exit door. Error rate was defined in terms of the number of decision points where the subject had to go in one direction or another along the hallway or turn a corner. The "Full Route" had six such decision points. The "Half Route" had four. An error was recorded whenever the subject was observed to have walked in the wrong (unintended) direction, turned the wrong way or hit a barrier. Post-trial debriefings were done to explore with subjects the strategies they had used to find the exit and also their preferences for and acceptance of the three different sounder guidance concepts.

Test Protocol. Twenty college students with normal hearing participated in the experiment. The test environment was the same as that used in the second pilot test and shown in Figure 4. The test protocol was also the same with the exception that subjects participated in trials using three sounder concepts rather than two.

Table 2. Total number of decision point errors in route following with various sounder patterns.

\begin{tabular}{|l|c|c|}
\hline \multirow{2}{*}{ Sounder Patterns } & \multicolumn{2}{c|}{ Route } \\
\cline { 2 - 3 } & Half Route & Full Route \\
\hline Sounders All @ Once & 5 & 6 \\
\hline Sequential Loop & 2 & 3 \\
\hline Single Sounder & 12 & 13 \\
\hline
\end{tabular}

*24 trials for each cell. 


\subsection{Results}

Errors. Table 2 shows the frequency of decision point errors across 24 trials in each condition. The frequency of errors was virtually the same in the Half Route and Full Route trials, so Chi Square analysis was performed on data collapsed over Route. A Chi Square analysis showed a significant effect of Sound Pattern on decision point errors $\left(x^{2}=15.4, \mathrm{df}=2, \mathrm{p}<0.00045\right)$.

Time of Completion. Table 3 shows the results for time to complete each egress trial with the various sounder patterns and with the Full Route and Half Route starting points. Analysis of Variance found a significant effect of Sounder Pattern $(\mathrm{F}=4.764$, $\mathrm{df}=46, \mathrm{p}<0.014)$ and of Route $(\mathrm{F}=45.544, \mathrm{df}=23, \mathrm{p}<0.0001)$. The interaction between Sounder Pattern and Route was not significant. Multiple comparisons between Sounder Patterns collapsed across route showed that time to complete egress was faster with both Single Sounder and Sequential Loop patterns than with the Sounder All @ Once pattern. Time of egress did not differ significantly between Single Sounder and Sequential Loop.

Table 3. Mean time (sec.) to complete a single egress trial with various sounder patterns and two different starting points.

\begin{tabular}{|l|c|c|}
\hline \multirow{2}{*}{ Sounder Patterns } & \multicolumn{2}{c|}{ Route } \\
\cline { 2 - 3 } & Half Route & Full Route \\
\hline Sounders All @ Once & 69.34 & 95.34 \\
& $(28.80)$ & $(29.62)$ \\
\hline Sequential Loop & 53.81 & 88.88 \\
& $(13.65)$ & $(17.25)$ \\
\hline Single Sounder & 53.00 & 94.26 \\
& $(8.83)$ & $(26.59)$ \\
\hline
\end{tabular}

$* 24$ trials for each cell.

Debriefing Results. At the conclusion of their test trials, subjects were debriefed and asked to provide ratings in response to several questions related to their experiences using the sounders for guidance and their preferences.

- Sounder pattern preference- Subjects' ranking of the three sounder patterns in order of preference revealed a significant preference for Sequential Loop over both the Sounders All @ Once and Single Sounder patterns $(\mathrm{p}<$ 0.01 for both comparisons by Wilcoxon Test) and for Sounder All @ Once over the Single Sounder pattern ( $\mathrm{p}<0.05$ by Wilcoxon Test).

- Egress strategy- Subjects were asked if they completely followed the sounder guidance or only partially followed the sounds, relying on other cues such as feeling the walls, barriers, etc. $83 \%$ indicated that they completely followed the sounder guidance in their Sequential Loop trials, compared to 79\% for the Sounder All @ Once trials and 33\% for the Single Sounder trials. 


\section{Discussion}

The results of the pilot studies and formal experiment suggest that using broadband sound emitted in carefully designed patterns can indeed be an effective medium for emergency route guidance in low visibility conditions. All three patterns investigated here, Single Sounder, Sounders All@ Once, and Sequential Loop with Redundant Cues, were able to guide subjects through evacuation routes laid out in various ways. Subjects committed significantly more errors in trials with the Single Sounder and also expressed their lowest preference for the Single Sounder. Subjects had to be within audible range of the single sounder for it to be useful, and even then, sometimes had difficulty localizing it and following it. So, while it can be effective under some conditions, the Single Sounder may be best suited as a redundant cue emitted in combination with one of the multiple sounder patterns.

The results also showed that, in the multi-sounder concepts, discriminating between adjacent pulse rates is key to providing clarity of guidance. Changing the Off component of the pulse rate for the Mid2 sounder from its factory setting of $200 \mathrm{msec}$ to a new value of $300 \mathrm{msec}$ made it more distinct from the Mid1 sounder and provided better guidance to the subjects through the middle portion of the route. However, even with this change, egress times in the half route trials for the Sounder All @ Once pattern were longer than one would expect if one were simply walking twice the distance. Subjects reported that this was due to some initial hesitation about which of the two adjacent sounders to follow. This was less an issue when a clear and nonoverlapping sequence of sounds was provided. Even greater pulse rate separation than was used in our experiment would likely mitigate this problem and make the Sounder All@ Once concept even more effective.

The Sequential Loop With Redundant Cues pattern was the most preferred method for guidance and resulted in the fewest errors at decision points. It's sequential pattern eliminated interference from adjacent sounders and effectively "pointed the way." Also, it highlighted the potential for improving route guidance by adding redundant cues, in this case, using the number of pulses at each sounder to encode its position in the sequence. Future research should more fully explore such concepts.

This was an initial investigation of using sounders in combinations to create patterns for emergency route guidance. Many questions remain. For example, using verbal cues or messages along the route in combination with the broadband sound might reduce some of the ambiguity of sound alone. Verbal cues will have to be carefully engineered to minimize interference with the broadband sound being emitted. Another question that must eventually be addressed is that of route adaptation. In the future, using computer models, we will have the ability to predict smoke and heat propagation from a fire, and classify the safety of evacuation routes according to those predictions. That creates the possibility of dynamically adapting routes to the conditions of smoke and heat and ensuring that evacuees are always guided along safe routes. But how is a change in route communicated to them? Future research will have to address that. 


\section{References}

1. Withington, D.J.: The use of directional sound to improve the safety of auditory warnings. In: Proceedings of the XIVth Triennial Congress of the International Ergonomics Association \& 44th Annual Meeting of the Human Factors and Ergonomics Society, San Diego, pp. 726-729 (2000)

2. Withington, D.J.: Faster evacuation from ferries with sound beacons. Fire 30 (2000)

3. Withington, D.J., Smith, S.: "White Noise" can help save stricken firefighters. Fire 27 (2000) 